\begin{tabular}{|c|l|}
\hline Title & Evidence for the Single Site Quadrupolar Kondo Effect in the Dilute Non-Kramers System Y 1-xPrxIr2Zn20 \\
\hline Author(s) & Y anagisawa, T.; Hidaka, H.; A mitsuka, H.; Zherlitsyn, S.; W osnitza, J.; Y amane, Y.; Onimaru, T. \\
\hline Citation & $\begin{array}{l}\text { Physical Review Letters, 123(6), 067201 } \\
\text { https://doi.org/L0.1103/PhysRevLett.123.067201 }\end{array}$ \\
\hline Issue Date & 2019-08-06 \\
\hline Doc URL & http://hdl.handle.net/2115/75729 \\
\hline Rights & @2019 A merican Physical Society \\
\hline Type & article \\
\hline File Information & PhysRevLett.123.067201.pdf \\
\hline
\end{tabular}

Instructions for use 


\title{
Evidence for the Single-Site Quadrupolar Kondo Effect in the Dilute Non-Kramers System $\mathbf{Y}_{1-x} \operatorname{Pr}_{x} \operatorname{Ir}_{2} Z_{20}$
}

\author{
T. Yanagisawa, ${ }^{1}$ H. Hidaka, ${ }^{1}$ H. Amitsuka, ${ }^{1}$ S. Zherlitsyn, ${ }^{2}$ J. Wosnitza, ${ }^{2,3}$ Y. Yamane, ${ }^{4}$ and T. Onimaru ${ }^{4}$ \\ ${ }^{1}$ Department of Physics, Hokkaido University, Sapporo 060-0810, Japan \\ ${ }^{2}$ Hochfeld-Magnetlabor Dresden (HLD-EMFL) and Würzburg-Dresden Cluster of Excellence ct.qmat, \\ Helmholtz-Zentrum Dresden-Rossendorf, 01328 Dresden, Germany \\ ${ }^{3}$ Institut für Festkörper- und Materialphysik, TU Dresden, 01062 Dresden, Germany \\ ${ }^{4}$ Graduate School of Advanced Sciences of Matter, Hiroshima University, Higashi-Hiroshima 739-8530, Japan
}

(Received 20 February 2019; revised manuscript received 27 May 2019; published 6 August 2019)

\begin{abstract}
Acoustic signatures of the single-site quadrupolar Kondo effect in $\mathrm{Y}_{0.966} \mathrm{Pr}_{0.034} \mathrm{Ir}_{2} \mathrm{Zn}_{20}$ are presented. The elastic constant $\left(C_{11}-C_{12}\right) / 2$, corresponding to the $\Gamma_{3}(E)$-symmetry electric-quadrupolar response, reveals a logarithmic temperature dependence of the quadrupolar susceptibility in the low-magnetic-field region below $\sim 0.3 \mathrm{~K}$. Furthermore, the Curie-type divergence of the elastic constant down to $\sim 1 \mathrm{~K}$ indicates that the $\mathrm{Pr}$ ions in this diluted system have a non-Kramers ground-state doublet. These observations evidence the single-site quadrupolar Kondo effect, as previously suggested based on specificheat and electrical-resistivity data.
\end{abstract}

DOI: 10.1103/PhysRevLett.123.067201

The Kondo effect [1], in a broad sense, appears ubiquitously as a phenomenon not only in dilute magnetic alloys or dense Kondo systems but also in various fields of physics [2,3]. In particular, heavy-electron physics has been intensively studied based on the Fermi-liquid theory originally introduced by Landau [4], where a local Fermi-liquid state is generally realized due to the Kondo effect driven by local magnetic impurity. An unconventional Kondo effect with concomitant non-Fermi-liquid (NFL) behavior of the physical quantities has been discussed since the 1990s (see Ref. [5] for a review) and some of the compounds that evidence NFL behavior have been recognized to realize a new type of Kondo phenomena, which is caused by overscreening of the local multipolar moment via two or more channels, the so-called multichannel Kondo effect [6]. The two-channel version of the multichannel Kondo effect [7] is the so-called quadrupolar Kondo effect (QKE), which was theoretically proposed by Cox $[8,9]$.

The quadrupolar Kondo model, which is invoked by a well-separated non-Kramers doublet $\Gamma_{3}(E)$ ground state in cubic symmetry, predicts several NFL peculiarities at very low temperatures such as a temperature dependence of the normalized electrical resistivity $\rho / \rho_{0} \propto 1 \pm \sqrt{T}$ [10], the presence of a fractional residual entropy $S=$ $\frac{1}{2} R \ln 2$ at absolute zero, and a logarithmic temperature dependence of the specific heat divided by temperature $C / T$ and also, of the quadrupolar susceptibility $\chi_{\Gamma}$ well below the characteristic temperature [11]. In particular, the appearance of $-\ln T$ behavior in the $\Gamma_{3}$ quadrupolar susceptibility in the cubic system can be observed as $+\ln T$ decreasing (softening) of the elastic constant $\left(C_{11}-C_{12}\right) / 2$ by means of ultrasound.
Many experimental investigations have been conducted to test this promising theoretical scenario, especially for uranium-based candidate materials, which were considered to have quadrupolar degrees of freedom (d.o.f.) and obey single-site NFL behavior, such as cubic $\mathrm{Y}_{1-x} \mathrm{U}_{x} \mathrm{Pd}_{3}$ and $\mathrm{Th}_{1-x} \mathrm{U}_{x} \mathrm{Be}_{13}$, as well as tetragonal $\mathrm{Th}_{1-x} \mathrm{U}_{x} \mathrm{Ru}_{2} \mathrm{Si}_{2}$ [12-14]. However, it is still elusive whether the quadrupolar d.o.f. are involved in the NFL behavior found in these compounds, because no convincing evidence has been found with respect to the main cause of these exotic phenomena, i.e., the local quadrupolar response. Because of the duality of the $5 f$ electrons (being partially localized as well as itinerant) and experimental difficulties, a nonmagnetic doublet ground state of the U $5 f$-electron system is possibly inappropriate to corroborate the QKE.

Cubic Pr-based non-Kramers doublet systems, such as $\operatorname{PrInAg}{ }_{2}, \mathrm{PrPb}_{3}$, and $\mathrm{PrMg}_{3}$, have since been included as candidate materials for the QKE of the $\operatorname{Pr}^{3+}\left(4 f^{2}, J=4\right)$ state, which has paved the way to corroborate Cox's quadrupolar-Kondo scenario of the $\mathrm{U}^{4+}\left(5 f^{2}, J=4\right)$ state [15-19]. A new family of cubic compounds, $\operatorname{Pr} T_{2} X_{20}$ ( $T=$ transition metal, $X=\mathrm{Al}, \mathrm{Zn}$, and $\mathrm{Cd}$ ), has recently attracted considerable attention [20]. Figure 1 shows the crystal structure of the $\operatorname{Pr} T_{2} X_{20}$ compounds, which have the $\mathrm{CeCr}_{2} \mathrm{Al}_{20}$-type structure ( $\mathrm{Fd} \overline{3} \mathrm{~m}, O_{h}^{7}$, No. 227), where a Pr guest ion (on the site 8a with $\mathrm{T}_{\mathrm{d}}$ symmetry) is encapsulated in a highly symmetric atomic cage consisting of $X_{16}$, which forms a diamond structure in the unit cell [21,22].

In this cage-structured-compound family, the Pr tends to have a $\Gamma_{3}(E)$ doublet crystalline electric field $(\mathrm{CEF})$ ground state. In particular, the Pr dilute-limit $\mathrm{Y}_{1-x} \operatorname{Pr}_{x} \mathrm{Ir}_{2} \mathrm{Zn}_{20}$ system 


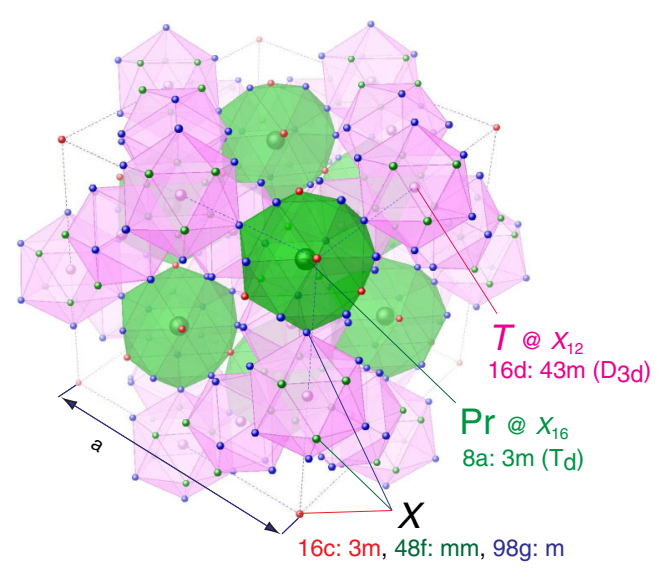

FIG. 1. Crystal structure of $\operatorname{Pr} T_{2} X_{20}$ ( $T=$ transition metal, $X=\mathrm{Al}, \mathrm{Zn}$, and $\mathrm{Cd}$ ). The Wycoff position and point-group symmetry of the atoms are also represented below each annotation.

is studied systematically to investigate a possible single-site quadrupolar Kondo state [23-25]. $\mathrm{Y}_{1-x} \mathrm{Pr}_{x} \mathrm{Ir}_{2} \mathrm{Zn}_{20}$ displays particular rich physics, ranging from a localized quadrupolar order of the $\Gamma_{3}(E)$ non-Kramers doublet ground state and superconductivity for $x=1$ [26-28] to possible single-site quadrupolar Kondo behavior for $x \rightarrow 0$, which appears as NFL behavior in the specific heat and resistivity. Here, we present data from ultrasonic measurements for the dilutelimit $\mathrm{Y}_{0.966} \mathrm{Pr}_{0.034} \mathrm{Ir}_{2} \mathrm{Zn}_{20} \quad(x=0.034)$. We find acoustic signatures of the $\Gamma_{3}(E)$ ground-state doublet, and a logarithmic temperature dependence of the $\Gamma_{3}(E)$-symmetry quadrupolar susceptibility, both of which give unambiguous evidence for the single-site quadrupolar Kondo effect.

Single crystals of $\mathrm{Y}_{1-x} \mathrm{Pr}_{x} \mathrm{Ir}_{2} \mathrm{Zn}_{20}$ were grown by $\mathrm{Zn}$-selfflux method with pre-arc-melting alloys of Y, Pr, and Ir as described in previous papers $[23,25]$. The Pr composition $x=0.034$ of the present sample is confirmed by the CEF analysis of the magnetization data, which is the same procedure used in the previous specific heat experiments [24]. The sample dimensions of the rectangular parallelepiped are $2.954 \times 2.771 \times 2.440 \mathrm{~mm}^{3}$ for [110]-[11̄0]-[001] Ultrasound is generated and detected by a pair of $\mathrm{LiNbO}_{3}$ transducers with a thickness of $100 \mu \mathrm{m}$, which were bonded on the sample surfaces with room-temperature-vulcanizing silicone. The quadrupolar responses can be observed as sound-velocity change by the phase-comparative method. The transverse sound velocity $v$ with propagation of $k \|$ [110] and polarization $u \|[1 \overline{1} 0]$ is converted to the elastic constant $C_{v}=\left(C_{11}-C_{12}\right) / 2\left(\mathrm{~J} / \mathrm{m}^{3}\right)$ by using the formula $C_{v}=\rho v^{2}$. Here, $\rho=8.277\left(\mathrm{~g} / \mathrm{cm}^{3}\right)$ is the density of the $x=0.034$ sample with the lattice constant $a=$ 14.1969(1) A. The low-temperature measurements were performed using a top-loading dilution refrigerator.

The temperature dependence of the relative change of the elastic constant $\left(C_{11}-C_{12}\right) / 2$ is displayed in Fig. 2. At $0 \mathrm{~T}$, we found Curie-like divergence (softening) of $\sim 0.18 \%$ in $\left(C_{11}-C_{12}\right) / 2$ below $2 \mathrm{~K}$ down to $0.04 \mathrm{~K}$.

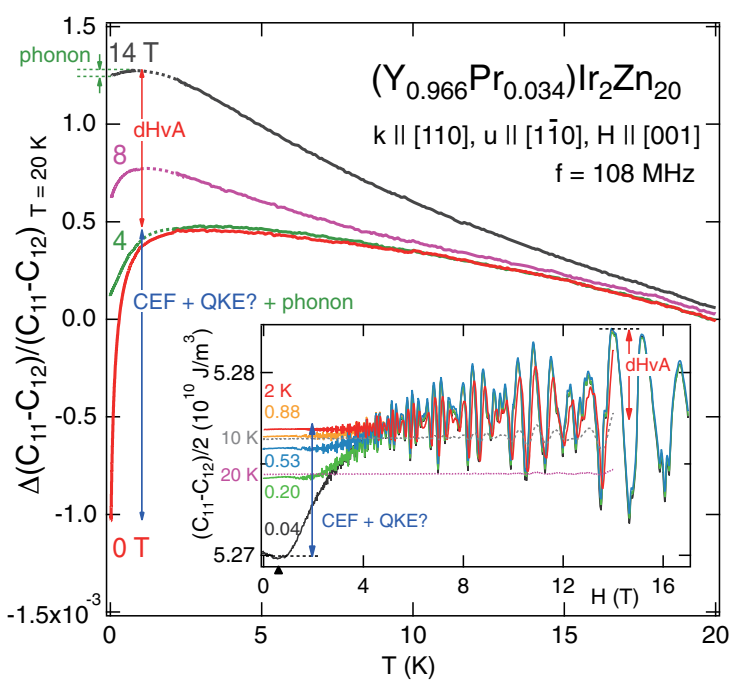

FIG. 2. Relative change of the elastic constant $\left(C_{11}-C_{12}\right) / 2$, as a function of temperature for various magnetic fields up to $14 \mathrm{~T}$ for $H \|[001]$ of $\mathrm{Y}_{0.966} \operatorname{Pr}_{0.034} \mathrm{Ir}_{2} \mathrm{Zn}_{20}$. The inset shows $\left(C_{11}-C_{12}\right) / 2$ as a function of magnetic field at various fixed temperatures between 0.04 and $20 \mathrm{~K}$.

The softening is reduced by an external magnetic field. At $14 \mathrm{~T}$, there remains a small magnetically robust softening of $\sim 0.02 \%$, which might be caused by quantum tunneling of $\mathrm{Zn}$ atoms at the site 16c and/or Pr and $\mathrm{Y}$ atoms at the site 8a, so-called, off-center tunneling (for details, see the Supplemental Material [29]) [30,31].

The temperature and magnetic-field dependence of the elastic constant corresponding to the point group symmetry $\Gamma$ in the local $4 f$-electron system can be described as a sum of three components of the elastic moduli, $C_{\Gamma}(T, H)=$ $C_{\Gamma}^{0}(T)+C_{\Gamma}^{4 f}(T, H)+C_{\Gamma}^{\mathrm{dHvA}}(T, H)$. Here, $C_{\Gamma}^{0}(T)$ is the temperature dependent (magnetic-field-independent) phonon background with the contribution from the off-center tunneling. $C_{\Gamma}^{4 f}(T, H)$ is the contribution from the $4 f$ electrons, mainly due to CEF effects, which can be described here by the quadrupolar susceptibility of the Pr ion and leads to the Curie-type softening [32]. $C_{\Gamma}^{\mathrm{dHvA}}(T, H)$ is the de Haasvan Alphen (dHvA) effect that leads to quantum oscillations in high-magnetic fields with frequencies proportional to $1 / H$ [33]. Since $C_{\Gamma}^{0}(T)$ is usually unaffected by magnetic field, we can simply estimate the latter two components in the above equation by comparing the $T$ and $H$ dependence of the elastic constant $C_{\Gamma 3}(T, H)=\left(C_{11}-C_{12}\right) / 2$.

The inset of Fig. 2 shows the magnetic-field dependence of the $\left(C_{11}-C_{12}\right) / 2$, where acoustic dHvA oscillations with frequencies of 148, 185, 332, and $295 \mathrm{~T}$ (decreasing order of oscillation amplitude) are clearly observed below $20 \mathrm{~K}$. Detailed analyses of these dHvA data are the subject of a forthcoming publication; however, this observation clearly proves the high quality of the present single crystal.

In the main panel of Fig. 2, the red, green, and blue double-headed arrows indicate the amount of change of 
$C_{\Gamma}^{\mathrm{dHvA}}$ for $14 \mathrm{~T}$, estimated from the field dependence, phonon contribution $C_{\Gamma}^{0}(T)$ below $1 \mathrm{~K}$, and the softening originated in $C_{\Gamma}^{0}(T)+C_{\Gamma}^{4 f}(T, H)$ for $0 \mathrm{~T}$ below $1 \mathrm{~K}$, respectively. Here, it is obvious that the major part of the low-temperature softening in the present compound can be interpreted by $C_{\Gamma}^{4 f}(T, H)$, which is a combination of CEF effects, nuclear effects, and a possible contribution from the QKE. Note that the temperature dependence of the dHvA oscillation amplitude $C_{\Gamma}^{\mathrm{dHvA}}(T)$ below $1 \mathrm{~K}$ is negligible compared to the other contributions because they only change within $\Delta C_{v} / C_{v} \sim 2 \times 10^{-5}$ for $3 \mathrm{~T}$ and $\sim 1 \times 10^{-4}$ for $13.5 \mathrm{~T}$. Based on the presence of the Curielike softening $\left(\propto T^{-1}\right)$ in $\left(C_{11}-C_{12}\right) / 2$ at low magnetic fields, it can be concluded that the Pr ions in the present dilute system also have a non-Kramers $\Gamma_{3}(E)$ CEF groundstate doublet, which is crucial for QKE. The amount of change in $\left(C_{11}-C_{12}\right) / 2$ in the present Y-rich system, $\Delta C_{v} / C_{v} \sim 0.18 \%$, is larger than the expected value $0.013 \%$ for $x=0.034$, which is simply estimated by multiplying the volume fraction of the Pr concentration to the softening of $0.39 \%$ observed at the antiferroquadrupolar ordering temperature $T_{\mathrm{Q}} \sim 0.11 \mathrm{~K}$ in the nondiluted compound $\left(\operatorname{PrIr}_{2} \mathrm{Zn}_{20}\right)[27,34]$. A possible reason for this discrepancy is the absence of the intersite quadrupolar-quadrupolar interactions in the present diluted system (as described later), since the amount of softening of the elastic constant will depend on the trade-off between the enhancement of the softening due to the volume fraction of the Pr ion, and the reduction of the softening due to the antiferrotype of the intersite quadrupolar-quadrupolar interactions. Further investigations of this system toward a wide range of $\operatorname{Pr}$ concentrations are needed to check a possible systematic change of the softening and the intersite quadrupolar interaction.

In Fig. 3 , the elastic constant $\left(C_{11}-C_{12}\right) / 2$ is represented as a function of temperature in logarithmic scale at various magnetic fields. Here, the contributions from $\mathrm{dHvA}$ effect below $1 \mathrm{~K}$ are estimated from Fig. 2 which is already subtracted as a constant background. Dotted and solid curves are the calculated quadrupolar susceptibility based on the local $4 f$-electron state of $\operatorname{Pr}^{3+}$ with and without the ${ }^{141}$ Pr's nuclear dipolar contribution, respectively. From the analysis, we notice that the local $4 f$-electron model well reproduces the data in $3 \mathrm{~T}$ and above, however, not below $3 \mathrm{~T}$ and $T<T^{*} \sim 1 \mathrm{~K}$, where the expected softening of $\left(C_{11}-C_{12}\right) / 2$ is strongly suppressed and also exhibits a logarithmic temperature dependence below $T_{0} \sim 0.3 \mathrm{~K}$. Here, the characteristic temperature $T_{0}$ is estimated from previous specific-heat measurements [25]. Based on the similarity of the logarithmic temperature dependence found in the specific heat and elastic constant, we may assume that there is a strong correlation between the NFL behavior and the quadrupolar fluctuations in the present system.

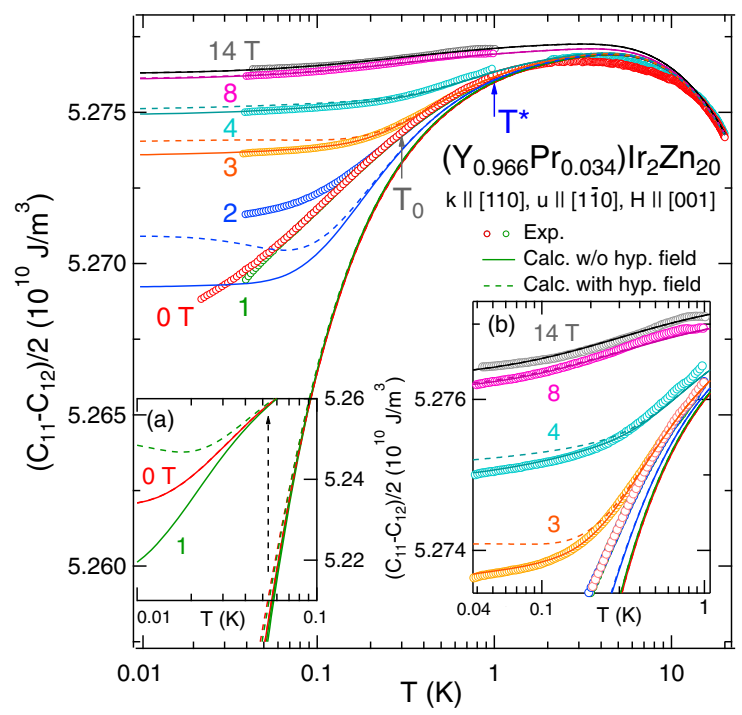

FIG. 3. Elastic constant $\left(C_{11}-C_{12}\right) / 2$ (open symbols) of $\mathrm{Y}_{0.966} \mathrm{Pr}_{0.034} \mathrm{Ir}_{2} \mathrm{Zn}_{20}$ as a function of temperature at various magnetic fields $H \|[001]$. Dashed and solid curves indicate the calculated quadrupolar susceptibility with and without the ${ }^{141} \operatorname{Pr}$ 's nuclear dipolar contribution, respectively (see text). The insets show enlargements of (a) the calculations below $0.1 \mathrm{~K}$ for 0 and $1 \mathrm{~T}$, and (b) the data above $3 \mathrm{~T}$.

Since we found no obvious frequency dependence of the ultrasound velocity in the range from 65 to $230 \mathrm{MHz}$ (see Supplemental Material [29]) and also no considerable ultrasonic attenuation at around $T_{0}$, a dynamical quadrupolar effect can be neglected, at least in the present frequency range. Thus, we analyze the elastic softening of $\left(C_{11}-C_{12}\right) / 2$ using the following formula describing the static quadrupolar susceptibility: $C_{\Gamma 3}^{4 f}(T, H)=C_{\Gamma 3}^{0}(T)-$ $N g_{\Gamma 3}^{2} \chi_{\Gamma 3}(T, H) /\left\{1-g_{\Gamma 3}^{\prime} \chi_{\Gamma 3}(T, H)\right\}$ (see the Supplemental Material [29] and Ref. [35]). Here, $C_{\Gamma 3}^{0}(T)$ is a magnetically independent background term and $N=0.095 \times$ $10^{27} \mathrm{~m}^{-3}$ is the number of Pr ions per unit volume in the $x=0.034$ sample estimated from the lattice constant at room temperature. $g_{\Gamma 3}=19.0 \mathrm{~K}$ is the coupling constant of the quadrupole-strain interaction, which is obtained from the best fit for the data above $3 \mathrm{~T}$, where the nonmagnetic Kramers doublet obeys Zeeman splitting and mixing with the wave function from the first excited $\Gamma_{4}\left(\mathrm{~T}_{1}\right)$ triplet CEF state (at $\sim 25.3 \mathrm{~K}$ in zero magnetic field). The coupling constant of intersite quadrupolar interaction is

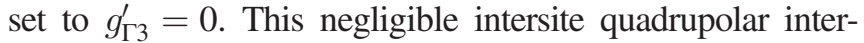
action, as obtained from our data for the diluted system, allows us to discuss the following single-site quadrupolar effect.

The $\Gamma_{3}(E)$-symmetry quadrupolar susceptibility $\chi_{\Gamma 3}(T, H)$ for $H \|[001]$ is calculated by using the quadrupolar operator $O_{2}^{2}=J_{x}^{2}-J_{y}^{2}$ and the previously reported CEF parameters for $\operatorname{PrIr}_{2} \mathrm{Zn}_{20}, B_{4}^{0}=-0.0109 \mathrm{~K}, B_{4}^{4}=5 B_{4}^{0}$, $B_{6}^{0}=-0.4477 \mathrm{mK}$, and $B_{6}^{4}=-21 B_{6}^{0}$ (equivalent to $W=$ $-1.219 \mathrm{~K}$ and $X=+0.537[22,36])$. In the present setup, the 


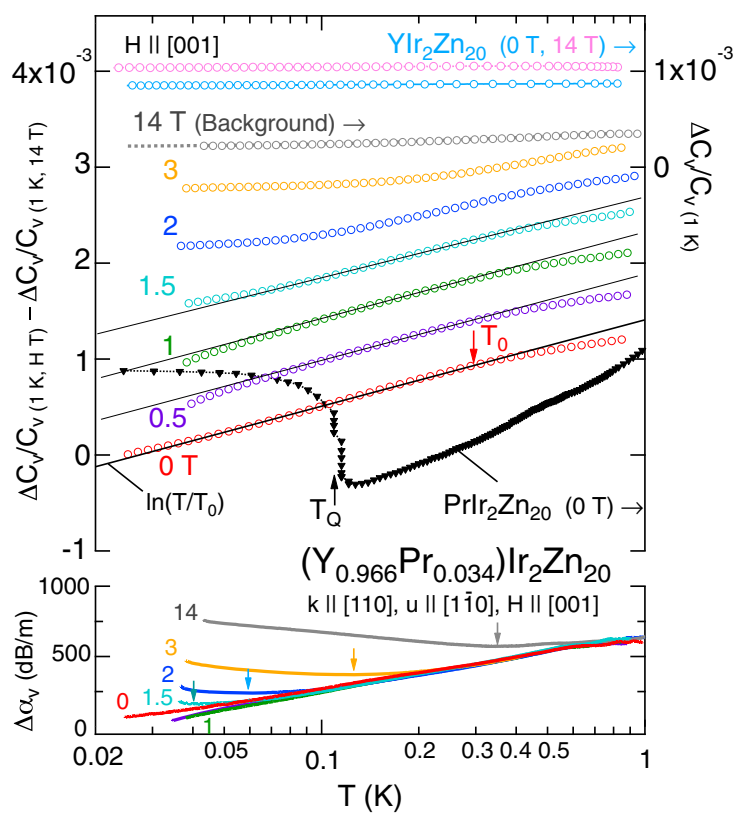

FIG. 4. (Upper panel) Temperature dependence of the background-subtracted elastic constant $\left(C_{11}-C_{12}\right) / 2$, shown as relative change, at selected magnetic fields. Data of $\operatorname{PrIr}_{2} \mathrm{Zn}_{20}$ $(x=1)$ [27] and $\mathrm{YIr}_{2} \mathrm{Zn}_{20}(x=0)$ are also displayed for comparison. The data are vertically shifted to facilitate display of the temperature and magnetic field variations. (Lower panel) Ultrasonic attenuation coefficient vs temperature.

rotationally invariant magnetoelastic effect [37] can be neglected. Note that a tiny tetragonal distortion of $B_{2}^{0}=$ $+0.003 \mathrm{~K}$ is added for the present calculation to reproduce the local minimum appearing at $\sim 0.7 \mathrm{~T}$ in the magnetic-field dependence of $\left(C_{11}-C_{12}\right) / 2$ at $0.04 \mathrm{~K}$ (arrowhead in the inset of Fig. 2). This may be caused by small inhomogeneities leading to a tiny disorder. This splits the $\Gamma_{3}(E)$ doublet into two singlets with a gap of $\Delta_{\Gamma 3}=0.048 \mathrm{~K}$, which could also be responsible for the deviation from the $+\ln T$ dependence below $\sim 0.06 \mathrm{~K}$ at $0.5-1.5 \mathrm{~T}$, as shown below in Fig. 4. This small gap obeys Zeeman splitting in magnetic field and a recovery of the degeneracy should occur in the vicinity of $0.6 \mathrm{~T}$ for $H \|[001]$. From these facts, it can be concluded that the present sample has a finite volume fraction of disordered Pr sites where the $4 f$ electrons are localized even in the NFL region. We also tested a random two-level (RTL) model [25] with a normal distribution of the gap value by assuming $0 \leq$ $\Delta_{\Gamma 3} \leq 0.096 \mathrm{~K}\left(0 \leq B_{2}^{0} \leq+0.006 \mathrm{~K}\right)$ with a mean value of $B_{2}^{0}=+0.003 \mathrm{~K}$. This RTL model does not reproduce the $+\ln T$ dependence of the elastic constant and further reveals the same result as the calculation done for a single mean value as shown by the solid curves in Fig. 3 .

We can also rule out the possibilities of the contributions from the nuclear spin $[38,39]$ and the effect of quantum tunneling of atoms (so-called off-center d.o.f. [30]) by following reasons. The dotted curves in Fig. 3 are the calculations including the additional contribution from hyperfine interactions of nuclear dipoles (rank 1) as described in the Supplemental Material [29]. The nuclear contribution causes a minor deviation below $\sim 0.1 \mathrm{~K}$ in the present analysis and makes the fit even worse. On the other hand, the effect of quantum tunneling of atoms can simply be estimated from the 14-T data, since it can be considered that the off-center d.o.f. must be magnetically robust. The background of the elastic constant $C_{\Gamma 3}^{0}(T)$ used in the present analysis is estimated from the fit to the whole temperature range of the data at $14 \mathrm{~T}$ (also see details in the Supplemental Material [29]). Indeed, the contribution from the off-center d.o.f. is relatively small compared to the change of the $+\ln T$ softening. According to the above considerations, we conclude that both of the nuclear dipolar (hyperfine) interaction and the off-center tunneling phenomena cannot reproduce the $+\ln T$ behavior in the elastic constant.

In Fig. 4, we replot the data on a logarithmic $T$ scale in which the 14-T data, representing the background of the off-center contribution, has been subtracted. The background contribution is negligibly small as shown in the 14-T data and $x=0\left(\mathrm{YIr}_{2} \mathrm{Zn}_{20}\right)$ system for comparison. It becomes obvious that the elastic constant $\left(C_{11}-C_{12}\right) / 2$ exhibits a $+\ln \left(T / T_{0}\right)$ dependence for temperature below $T_{0} \sim 0.3 \mathrm{~K}$ and below $1.5 \mathrm{~T}$. This logarithmic divergence of the quadrupolar susceptibility, i.e., logarithmic softening of the elastic constant, is consistent with the prediction from the quadrupolar Kondo theory [8,9], and also clearly identifies that the channel number of the Kondo effect in the present system must be two, in contrast to the singlechannel Kondo effect (magnetic dipolar in origin), which leads to a leveling-off feature of the quadrupolar susceptibility [7,11]. The small deviation below about $0.06 \mathrm{~K}$ $\left(\Delta_{\Gamma 3}\right)$ has been discussed above and is probably caused by tiny disorder. When applying magnetic field, the data deviate from the $+\ln \left(T / T_{0}\right)$ behavior and seem to cross over to localized electronic states above $3 \mathrm{~T}$. The fact is also consistent with the preconceived scenario that the Zeeman splitting of not only the $\Gamma_{3}(E)$ doublet of the local $4 f$ electrons, due to the mixing of the first excited $\Gamma_{4}\left(\mathrm{~T}_{1}\right) \mathrm{CEF}$ state, but also the splitting of the $\Gamma_{8}$ states of the conduction band in finite magnetic fields will destroy the $c-f$ hybridization, which was driving the QKE. The ultrasonic attenuation coefficient is displayed in the lower panel of Fig. 4. The upturn appearing towards low temperatures in the ultrasonic attenuation (indicated by down arrows) strongly suggests a mixing of the excited $\Gamma_{4}\left(\mathrm{~T}_{1}\right)$ triplet state in applied magnetic fields, while there is no enhancement or anomaly in the ultrasonic attenuation at fields below $1.5 \mathrm{~T}$, which also rules out dynamical effects, such as the vibronic state [40]. Based on the above investigations, the logarithmic temperature dependence of the elastic constant $\left(C_{11}-C_{12}\right) / 2$ is considered to be clearly and strongly relevant to the recent finding of the NFL behavior in the electrical resistivity and specific heat of $\mathrm{Y}_{0.966} \mathrm{Pr}_{0.034} \mathrm{Ir}_{2} \mathrm{Zn}_{20}$ 
in the diluted $\operatorname{Pr}$ systems in the same temperature and magnetic field region. Further investigations are needed to check a possible systematic change in the logarithmic behavior toward a wide range of Pr concentrations.

In conclusion, we have reported that the elastic constant $\left(C_{11}-C_{12}\right) / 2$, related to the $\Gamma_{3}(E)$-symmetry quadrupolar susceptibility, of $\mathrm{Y}_{0.966} \mathrm{Pr}_{0.034} \mathrm{Ir}_{2} \mathrm{Zn}_{20}$ obeys a Curie law down to $\sim 1 \mathrm{~K}$. $\left(C_{11}-C_{12}\right) / 2$ exhibits a strong deviation from the localized $4 f$-electron model and a logarithmic temperature dependence below $T_{0} \sim 0.3 \mathrm{~K}$, where NFL behavior in the specific heat and electrical resistivity have previously been reported. This logarithmic temperature variation manifested in the $\Gamma_{3}(E)$-symmetry quadrupolar susceptibility is consistent with the theoretical prediction of a QKE by Cox. Thus, it is reasonable to consider that the local NFL behavior of the present compound arises from a single-site two-channel (electric quadrupolar) Kondo effect.

The present research was supported by JSPS KAKENHI Grants No. JP15KK0169, No. JP18H04297, No. JP18H01182, No. JP17K05525, No. JP18KK0078, No. JP15KK0146, No. JP15H05882, No. JP15H05885, No. JP15H05886, No. JP15K21732, and the Strategic Young Researcher Overseas Visits Program for Accelerating Brain Circulation from JSPS. We acknowledge support from the DFG through the Würzburg-Dresden Cluster of Excellence on Complexity and Topology in Quantum Matter - ct.qmat (EXC 2147, project-id 39085490) and from the HLD at HZDR, member of the European Magnetic Field Laboratory (EMFL). T. Y. would like to thank J. Klotz for supporting the measurements at HZDR.

[1] J. Kondo, Prog. Theor. Phys. 32, 37 (1964).

[2] P. Coleman, in Introduction to Many Body Physics (Cambridge University Press, Cambridge, England, 2015).

[3] P. Coleman, in Handbook of Magnetism and Advanced Magnetic Materials, edited by H. Kronmüller and S. Parkin (John Wiley and Sons, Hoboken, New Jersey, 2007), Vol. 1, p. 95.

[4] L. D. Landau, Sov. Phys. JETP 3, 920 (1957).

[5] M. B. Maple, J. Phys. Soc. Jpn. 74, 222 (2005).

[6] P. Nozières and A. Blandin, J. Phys. 41, 193 (1980).

[7] P. D. Sacramento and P. Schlottmann, Phys. Rev. B 43, 13294 (1991).

[8] D. L. Cox, Phys. Rev. Lett. 59, 1240 (1987).

[9] D. L. Cox and A. Zawadowski, Adv. Phys. 47, 599 (1998).

[10] I. Affleck and A. W. W. Ludwig, Phys. Rev. B 48, 7297 (1993).

[11] H. Kusunose, J. Phys. Soc. Jpn. 85, 064708 (2016).

[12] C. L. Seaman, M. B. Maple, B. W. Lee, S. Ghamaty, M. S. Torikachvili, J. S. Kang, L. Z. Liu, J. W. Allen, and D. L. Cox, Phys. Rev. Lett. 67, 2882 (1991).

[13] F. G. Aliev, H. E. Mfarrej, S. Vieira, and R. Villar, Solid State Commun. 91, 775 (1994).
[14] H. Amitsuka and T. Sakakibara, J. Phys. Soc. Jpn. 63, 736 (1994).

[15] A. Yatskar, W. P. Beyermann, R. Movshovich, and P. C. Canfield, Phys. Rev. Lett. 77, 3637 (1996).

[16] E. Bucher, K. Andres, A. C. Gossard, and J. P. Maita, J. Low Temp. Phys. 2, 322 (1972).

[17] T. Onimaru, T. Sakakibara, N. Aso, H. Yoshizawa, H. S. Suzuki, and T. Takeuchi, Phys. Rev. Lett. 94, 197201 (2005).

[18] T. Kawae, K. Kinoshita, Y. Nakaie, N. Tateiwa, K. Takeda, H. S. Suzuki, and T. Kitai, Phys. Rev. Lett. 96, 027210 (2006).

[19] H. Tanida, H. S. Suzuki, S. Takagi, H. Onodera, and K. Tanigaki, J. Phys. Soc. Jpn. 75, 073705 (2006).

[20] T. Onimaru and H. Kusunose, J. Phys. Soc. Jpn. 85, 082002 (2016).

[21] T. J. Sato, S. Ibuka, Y. Nambu, T. Yamazaki, T. Hong, A. Sakai, and S. Nakatsuji, Phys. Rev. B 86, 184419 (2012).

[22] K. Iwasa, H. Kobayashi, T. Onimaru, K. T. Matsumoto, N. Nagasawa, T. Takabatake, S. Ohira-Kawamura, T. Kikuchi, Y. Inamura, and K. Nakajima, J. Phys. Soc. Jpn. 82, 043707 (2013).

[23] Y. Yamane, T. Onimaru, K. Uenishi, K. Wakiya, K. Matsumoto, K. Umeo, and T. Takabatake, Physica (Amsterdam) 536B, 40 (2018).

[24] Y. Yamane, T. Onimaru, K. Wakiya, K. T. Matsumoto, K. Umeo, and T. Takabatake, AIP Adv. 8, 101338 (2018).

[25] Y. Yamane, T. Onimaru, K. Wakiya, K. T. Matsumoto, K. Umeo, and T. Takabatake, Phys. Rev. Lett. 121, 077206 (2018).

[26] T. Onimaru, K. T. Matsumoto, Y. F. Inoue, K. Umeo, Y. Saiga, Y. Matsushita, R. Tamura, K. Nishimoto, I. Ishii, T. Suzuki, and T. Takabatake, J. Phys. Soc. Jpn. 79, 033704 (2010).

[27] I. Ishii, H. Muneshige, Y. Suetomi, T. K. Fujita, T. Onimaru, K. T. Matsumoto, T. Takabatake, K. Araki, M. Akatsu, Y. Nemoto, T. Goto, and T. Suzuki, J. Phys. Soc. Jpn. 80, 093601 (2011).

[28] K. Iwasa, K. T. Matsumoto, T. Onimaru, T. Takabatake, J. M. Mignot, and A. Gukasov, Phys. Rev. B 95, 155106 (2017).

[29] See Supplemental Material at http://link.aps.org/ supplemental/10.1103/PhysRevLett.123.067201 for further details on ultrasonic methods, calculations, and analyses of nuclear and quantum tunneling contributions, and de Haasvan Alphen oscillation.

[30] T. Goto, Y. Nemoto, T. Yamaguchi, M. Akatsu, T. Yanagisawa, O. Suzuki, and H. Kitazawa, Phys. Rev. B 70, 184126 (2004).

[31] K. Wakiya, T. Onimaru, S. Tsutsui, T. Hasegawa, K. T. Matsumoto, N. Nagasawa, A. Q. R. Baron, N. Ogita, M. Udagawa, and T. Takabatake, Phys. Rev. B 93, 064105 (2016).

[32] B. Lüthi, Physical Acoustics in the Solid State (Springer, Berlin, 2006).

[33] M. Kataoka and T. Goto, J. Phys. Soc. Jpn. 62, 4352 (1993).

[34] T. Onimaru, K. T. Matsumoto, Y. F. Inoue, K. Umeo, T. Sakakibara, Y. Karaki, M. Kubota, and T. Takabatake, Phys. Rev. Lett. 106, 177001 (2011). 
[35] T. Yanagisawa, S. Mombetsu, H. Hidaka, H. Amitsuka, P. T. Cong, S. Yasin, S. Zherlitsyn, J. Wosnitza, N. K. K. Huang, M. Janoschek, M. B. Maple, and D. Aoki, Phys. Rev. B 97, 155137 (2018).

[36] K. R. Lea, M. J. M. Leask, and W. P. Wolf, J. Phys. Chem. Solids 23, 1381 (1962).

[37] V. Dohm and P. Fulde, Z. Phys. B 21, 369 (1975).
[38] Y. Aoki, T. Namiki, T. T. S. R. Saha, T. Sakakibara, R. Shiina, H. Shiba, H. Sugawara, and H. Sato, J. Phys. Soc. Jpn. 80, 054704 (2011).

[39] J. Kondo, J. Phys. Soc. Jpn. 16, 1690 (1961).

[40] K. Araki, T. Goto, K. Mitsumoto, Y. Nemoto, M. Akatsu, H. S. Suzuki, H. Tanida, S. Takagi, S. Yasin, S. Zherlitsyn, and J. Wosnitza, J. Phys. Soc. Jpn. 81, 023710 (2012). 